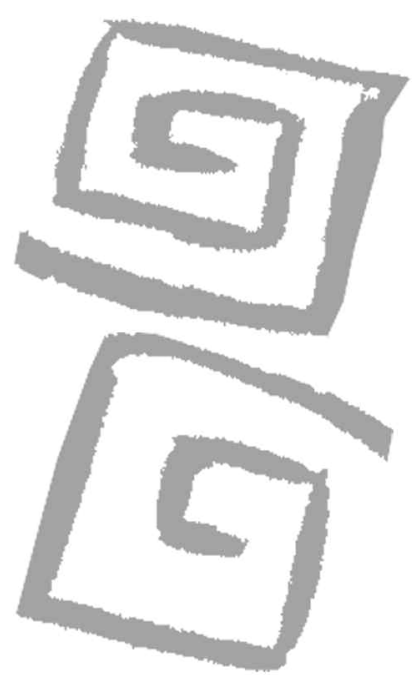

\title{
Análisis de los motivos del abandono del proceso de seguimiento y tratamiento por parte de mujeres con lesiones precursoras de cáncer de cuello uterino en la provincia de Jujuy: implicancias para la gestión
}

\author{
Analysis of the reasons for abandoning the follow-up \\ and treatment process in women with pre-cancerous \\ cervical lesions in the province of Jujuy: implications \\ for health management
}

Paolino, Melisa ${ }^{1}$; Arrossi, Silvina ${ }^{2}$

\footnotetext{
${ }^{1}$ Licenciada en Sociología. Investigadora becaria, Consejo Nacional de Investigaciones Científicas y Técnicas (CONICET), Centro de Estudios de Estado y Sociedad (CEDES), Buenos Aires, Argentina. melisa@cedes.org

${ }^{2}$ Licenciada en Sociología. Docteur en Demographie. Investigadora Independiente, Consejo Nacional de Investigaciones Científicas y Técnicas (CONICET). Investigadora Titular, Centro de Estudios de Estado y Sociedad (CEDES), Buenos Aires, Argentina. silviarrossi2020@gmail.com
}

RESUMEN El adecuado seguimiento y tratamiento de las mujeres con lesiones escamosas intraepiteliales de alto grado (HSIL, por sus siglas en ingles) y carcinoma es un aspecto clave para la reducción de la mortalidad por cáncer de cuello uterino. El objetivo de esta investigación es analizar la magnitud y los motivos del abandono en el proceso de seguimiento y tratamiento por parte de las mujeres con diagnóstico de HSIL y carcinoma atendidas en el sistema público de la provincia de Jujuy durante 2010. Se realizó el análisis de fuentes secundarias y entrevistas domiciliarias a las mujeres. El porcentaje de abandono en la población de estudio fue del 23,5\%. Entre estas mujeres, el 40,5\% mencionó motivos de abandono relacionados con la organización del sistema de salud y el 30,9\% mencionó motivos subjetivos. Los resultados han sido utilizados como insumos para la reorganización del proceso de seguimiento y tratamiento que se está llevando a cabo en la provincia.

PALABRAS CLAVE Neoplasias del Cuello Uterino; Papanicolaou; Prevención de Cáncer de Cuello Uterino; Desistencia al Tratamiento; Argentina.

\begin{abstract}
Adequate follow-up and treatment of women with high-grade squamous intraepithelial lesion (HSIL) and cancer is key in reducing cervical cancer mortality. This research study aims to analyze the magnitude of abandonment in the follow-up and treatment process, as well as the reasons for this abandonment, in women with HSIL who received care in public health services in the province of Jujuy in 2010. Secondary sources were analyzed and women were interviewed in their homes. The abandonment rate within the study population was $23.5 \%$. Among these women, $40.5 \%$ mentioned organizational problems in the health care system as a reason for abandoning and $30.9 \%$ mentioned subjective reasons. Our results have been used in the reorganization of the follow-up and treatment process being carried out in the province.
\end{abstract}

KEY WORDS Uterine Cervical Neoplasms; Papanicolaou Smear; Cervix Neoplasm Prevention; Patient Dropouts; Argentina. 


\section{INTRODUCCIÓN}

El cáncer de cuello uterino es uno de los pocos cánceres que puede ser prevenido con la tecnología actualmente disponible. Se ha demostrado que el uso de la prueba de Papanicolaou (Pap), en el marco de programas organizados de prevención (a), ha logrado reducir la mortalidad por esta enfermedad en un $80 \%$ (1). En los últimos años, el descubrimiento de la asociación causal entre el virus del papiloma humano (VPH) y el cáncer de cuello uterino $(2,3)$ ha permitido el desarrollo de nuevas tecnologías (test de ADN-VPH y la vacuna contra el VPH) que han abierto nuevas perspectivas para la prevención. Por ejemplo, la mayor sensibilidad y el mayor valor predictivo negativo del test de VPH con respecto a la citología reducen la cantidad de tamizajes que la mujer debe realizar (4-6).

Sin embargo, el desarrollo de la tecnología no reduce por sí mismo la incidencia y mortalidad por este tumor que afecta mayormente a las mujeres pobres, con menor nivel educativo y con menor acceso a los servicios de salud $(7,8)$. En Argentina, durante el año 2010, murieron aproximadamente 1.800 mujeres a causa del cáncer de cuello uterino -tasa de mortalidad estandarizada por edad (TME) 7 por 100.000 mujeres- (9). Asimismo, la distribución de la mortalidad en nuestro país es extremadamente desigual: las TME de las provincias más pobres como Misiones (17 por 100.000) o Jujuy (12 por 100.000) son 3 o 4 veces más altas que la de la Ciudad Autónoma de Buenos Aires (4 por 100.000), la región más rica del país (9). La alta mortalidad está vinculada a fallas en la continuidad del proceso de prevención, en el que el test de tamizaje es solo el componente tecnológico. En efecto, el proceso de prevención del cáncer de cuello uterino está constituido por cuatro etapas fundamentales: el tamizaje (b) -mediante el Pap, la prueba de VPH, etc.-; el diagnóstico -biopsia, etc.-; el tratamiento -procedimiento de escisión electroquirúrgica con asa (LEEP, por sus siglas en inglés) (c)-; y el seguimiento posterior al tratamiento.

Las etapas posteriores al tamizaje son críticas para la prevención de esta enfermedad. Un estudio realizado en Colombia mostró que un seguimiento y tratamiento adecuados tienen más impacto en la reducción de la mortalidad que la alta cobertura de tamizaje: el seguimiento y el tratamiento del $50 \%$ de la población con lesiones precancerosas en un contexto de $100 \%$ de cobertura de tamizaje pueden reducir el riesgo de mortalidad al 50\%, mientras que el $100 \%$ del seguimiento y el tratamiento en un contexto de $50 \%$ de cobertura de tamizaje pueden reducirlo en alrededor de un $70 \%$ (10).

A pesar de su relevancia en la prevención del cáncer de cuello uterino, en Argentina existe un enorme vacío de conocimiento en cuanto a cuál es la magnitud del abandono del proceso de seguimiento y tratamiento y sus factores explicativos subyacentes. Un diagnóstico de situación del Programa Nacional de Prevención de Cáncer Cérvico-uterino y de los programas provinciales en Argentina, Ilevado a cabo en 2007-2008 (11), mostró que la mayor parte de los programas provinciales no tenía registro de cuántas mujeres con lesiones precancerosas habían continuado el proceso de seguimiento y tratamiento. En la gran mayoría de los establecimientos de salud tampoco era posible conseguir esa información dado que no eran datos que se registraran sistemáticamente. La ausencia de registros también fue relevada en servicios públicos de salud de un municipio del Conurbano bonaerense (12). El único antecedente en la Argentina es una investigación realizada a fines de la década de 1980 en cuatro hospitales de la provincia de Buenos Aires (13), que mostró que el $40 \%$ de las mujeres entrevistadas con citología anormal había abandonado todo curso de acción con relación a su enfermedad.

Con el fin de cubrir estas áreas de vacancia, desde el año 2010 nuestro equipo ha desarrollado distintas investigaciones (d) en el Conurbano bonaerense y provincias seleccionadas cuyo objetivo principal ha sido analizar la magnitud del abandono y las dimensiones socioeconómicas, institucionales y simbólicas que influyen en el proceso de seguimiento y tratamiento de mujeres con HSIL y carcinoma. Como parte de este proyecto más amplio entre noviembre de 2010 y julio de 2011 se llevó a cabo en la provincia de Jujuy una investigación cuyos objetivos fueron: 
1) describir la magnitud del abandono en el proceso de seguimiento y tratamiento de las mujeres con HSIL y carcinoma.

2) analizar las características socioeconómicas de las mujeres y su relación con el abandono del proceso de atención;

3) identificar los motivos de abandono;

4) analizar la calidad de la entrega de los resultados desde la perspectiva de las mujeres y su relación con el abandono del proceso de atención;

5) elaborar una propuesta conjunta con los efectores locales para el fortalecimiento del proceso de seguimiento y tratamiento de mujeres con HSIL y carcinoma.

Durante el proyecto se realizó un trabajo articulado con los efectores locales (el Programa Provincial de Prevención y Control de Cáncer de Cuello Uterino y Cáncer de Mamas, la Dirección Provincial de APS y Acción Comunitaria, el Servicio de Anatomía Patológica y el Servicio de Patología Cervical del hospital provincial de referencia) no solo para acceder a los datos disponibles, sino también para facilitar la aplicación de los resultados de la investigación y contribuir con el mejoramiento de los procesos de atención. El proyecto fue realizado con el apoyo del Programa Nacional de Prevención de Cáncer Cérvico-uterino y del Programa Provincial de Prevención y Control de Cáncer de Cuello Uterino y Cáncer de Mamas de la provincia de Jujuy, y fue financiado por la Organización Panamericana de la Salud (OPS).

En este artículo presentamos los resultados de este proyecto que según nuestro conocimiento es la primera investigación sobre la magnitud y los motivos de abandono del proceso de seguimiento y tratamiento de mujeres con HSIL y carcinoma atendidas en la totalidad de los efectores públicos de salud de la provincia de Jujuy. Sus resultados han sido tomados como insumos para la reorganización del sistema de referencia y contrarreferencia que se está llevando a cabo en esa provincia.

\section{METODOLOGÍA}

\section{Ámbito de realización}

Se seleccionó la provincia de Jujuy dado que es una de las provincias consideradas como prioritaria por el Programa Nacional debido a su alta tasa de mortalidad. Por otro lado, se contaba con el apoyo de las autoridades provinciales, quienes demandaban información que les permitiera medir la amplitud del problema y delinear soluciones para resolverlo.

El sistema de salud público jujeño cuenta con aproximadamente 250 centros de Atención Primaria de la Salud (CAPS) en donde se realiza la toma de Pap (e), cuatro laboratorios de citología; un servicio de anatomía patológica de referencia; nueve centros de diagnóstico (en donde se realiza colposcopía y biopsia) y cinco servicios en donde se realiza el tratamiento. El tamizaje, diagnóstico y tratamiento están disponibles de forma gratuita en el sistema de salud público provincial.

\section{Criterios de inclusión/exclusión de la muestra}

La población incluida en el estudio fue el universo de mujeres mayores de 18 años atendidas, entre el 1 de enero y el 31 de diciembre de 2010, en instituciones de salud públicas de la provincia de Jujuy y que registraban los siguientes diagnósticos según la clasificación Bethesda de citologías (15): células escamosas atípicas de significado indeterminado sugestivo de lesión intraepitelial de alto grado (ASC-H por sus siglas en inglés), lesión escamosa intraepitelial de alto grado (HSIL por sus siglas en inglés) y carcinoma. Se seleccionaron dichos diagnósticos por ser lesiones precursoras de cáncer de cuello uterino, para las que está indicada la realización de biopsia y eventual tratamiento (16). Fueron excluidas del estudio las mujeres menores de 18 años, las mujeres que registraban algún Pap HSIL o superior previo al año 2010 o bien habían recibido tratamiento antes del año 2010.

\section{Definición de abandono del proceso de atención}

Según Ramos y Pantelides (13) el abandono del proceso de atención supone que la mujer, en algún punto de la trayectoria que se inicia a partir de la realización del Pap, deja de concurrir al 
servicio de origen y no concurre a ningún otro servicio. Considerando el último episodio registrado, el abandono es definido según el tipo de intervención médica realizada y el tiempo transcurrido desde entonces, como se detalla a continuación:

1) abandono en la etapa de tamizaje: más de 60 días desde la toma del Pap inicial sin retirar/conocer el resultado;

2) abandono en la etapa de diagnóstico: más de 90 días entre la entrega de resultado y la realización de la biopsia;

3) abandono en la etapa de tratamiento: más de 90 días entre la realización de la biopsia y la realización de tratamiento.

\section{Técnicas de recolección de datos}

\section{Fuentes secundarias: sistematización de regis- tros disponibles}

Entre el 6 de diciembre de 2010 y el 1 de marzo de 2011, siguiendo el modelo de estudios consultados $(13,17,18)$, se realizó la recopilación, sistematización y análisis de la información de diversas fuentes secundarias disponibles.

La provincia cuenta con el Sistema de Información para el Tamizaje (SITAM 1.0) implementado por el Programa Nacional, que permite llevar el registro en línea de los procedimientos realizados por los centros de toma de muestras, los laboratorios y las unidades de tratamiento, facilitando el seguimiento de las mujeres en las distintas etapas del proceso de atención (9). Si bien este sistema es utilizado en la provincia desde 2009, la información sobre biopsias y tratamientos no se registraba en el momento de la investigación de manera completa. Por lo tanto, fue necesaria la recopilación de datos en otras fuentes: registros de laboratorios de citología y anatomía patológica y libros de biopsias y tratamientos de los servicios de patología cervical. Los datos básicos de las mujeres y el proceso de atención desde el diagnóstico de Pap HSIL en adelante se transcribieron a una base de datos única considerando las siguientes variables: fechas de cada contacto de la mujer con el sistema de salud en torno a este tema; procedimientos (repetición de Pap, colposcopia, biopsia, tratamiento aplicado); y resultados de cada uno de los procedimientos realizados.

Las variables utilizadas para compatibilizar los registros y asegurar que los datos acerca de la trayectoria de atención correspondían a la misma mujer fueron el número de Documento Nacional de Identidad, cédula o número de historia clínica, apellido y nombre. En caso de duplicaciones o inconsistencias en los diagnósticos y/o datos personales se contrastó la información con los informes de citología disponibles en los archivos del laboratorio del hospital de referencia.

\section{Fuentes primarias: encuestas a las mujeres}

Una vez obtenida la base de datos única, fue posible identificar a las mujeres que no registraban información acerca de la realización de procedimientos de diagnóstico y tratamiento. Sin embargo, esta información no permitía determinar si la falta de datos se debía al abandono del proceso de atención, a un problema de registro o bien a que la mujer había continuado su atención en otro subsector del sistema de salud (obra social, seguro de salud privado). Es por esto que fue necesaria la realización de encuestas a las mujeres que no registraban información sobre diagnóstico y/o tratamiento para contrastar la información obtenida en los registros, cuantificar el abandono y describir la trayectoria de atención de manera más precisa.

Se realizaron encuestas domiciliarias utilizando un cuestionario estructurado. Las variables sociodemográficas y económicas incluidas fueron:

- edad en años cumplidos;

- máximo nivel educativo alcanzado: terciario incompleto/secundario completo, secundario incompleto/primario completo, primario incompleto/nunca concurrió a la escuela;

- situación familiar: con pareja e hijos, con pareja sin hijos, sin pareja con hijos, sin pareja sin hijos;

- jefatura del hogar: sí/no;

- situación ocupacional: ocupada, desocupada, ama de casa (dedicada a la gestión del hogar y cuidado de personas dependientes), inactiva (estudiante, jubilada, pensionada, permanentemente discapacitada, excluyendo la dedicación a las tareas del hogar); 
- situación habitacional: a) inadecuada (viviendas con al menos una de estas características: sin agua por cañerías dentro de la vivienda, con letrina, con piso de tierra o con presencia de hacinamiento -más de tres personas por cuarto, excluyendo baño y cocina (19)- y b) adecuada (viviendas que no cumplen con ninguna de esas condiciones).

Las variables incluidas para medir el uso del sistema de salud fueron: tipo de cobertura de salud (pública, privada/obra social); y frecuencia de visita al ginecólogo (cada 3/6/12 meses; en los embarazos, no tiene frecuencia).

La reconstrucción del proceso de atención desde el Pap en adelante fue realizada mediante preguntas abiertas que indagaban acerca de las instituciones a las cuales concurrió la mujer, los procedimientos recomendados por los profesionales, los procedimientos efectivamente realizados y sus resultados.

Para analizar la calidad percibida en la entrega del resultado del Pap, se preguntó a las mujeres si conocían su resultado, el tiempo entre la realización del Pap y la recepción del resultado y la manera en la que fue entregado (en la consulta, mediante un Ilamado telefónico, visita domiciliaria). Adicionalmente, a las mujeres que conocían el resultado $(n=71)$, se les formularon las siguientes preguntas: ¿Le explicaron lo que significaba el resultado? (sí/no); ¿Le explicaron cuáles eran los pasos que debía realizar posteriormente? (sí/no); y ¿El médico le preguntó si tenía alguna consulta o duda? (sí/no). Las respuestas afirmativas a estas preguntas se consideraron indicadores de buena calidad de entrega de resultado percibida por las mujeres.

A las mujeres que habían abandonado el proceso de atención se les preguntó sobre el principal motivo de abandono. Los motivos fueron recategorizados según criterios utilizados en estudios previos (20-22):

1) Motivos relacionados con la organización y funcionamiento del sistema de salud.

1.1) Motivos relativos a la organización de los servicios (atrasos en la entrega de resultados, dificultades para obtener turnos, suspensión en la atención y largos tiempos de espera para la consulta).
1.2) Motivos relacionados con el proceso de atención (problemas en la comunicación entre médico y mujeres y malas experiencias en la atención).

2) Motivos subjetivos (miedo, fatalismo, negación, no considerar necesario volver a la consulta).

3) Motivos personales/familiares (problemas de pareja, separaciones, fallecimiento de hijos).

4) Motivos laborales (no poder faltar al trabajo).

5) Motivos relativos a la carga de trabajo doméstico (cuidado de los hijos u otro familiar).

6) Motivos relativos al traslado (falta de dinero para el traslado y lejanía).

Las encuestas fueron realizadas por dos encuestadoras locales previamente capacitadas. Una vez finalizada la entrevista, se entregó a las mujeres material informativo sobre prevención de cáncer de cuello uterino y una nota de derivación con datos de las instituciones y profesionales que podían contactar para continuar con el proceso de atención. La red de derivación fue consensuada previamente con los efectores locales para asegurar la revinculación de las mujeres con los servicios de salud en caso de abandono.

Se obtuvo la firma del consentimiento informado de todas las entrevistadas y el proyecto fue aprobado por el Comité de Bioética Provincial dependiente del Ministerio de Salud de la Provincia de Jujuy. En todos los casos se ha respetado la confidencialidad de los datos y la identidad de las mujeres entrevistadas.

\section{Análisis de datos}

El procesamiento y análisis de datos se realizó mediante el sistema estadístico STATA 10.0. Se realizó un análisis univariado y bivariado, elaborando cuadros de frecuencias y porcentajes para cada una de las variables del estudio. Para evaluar la relación entre las variables sociodemográficas, económicas y de calidad de la entrega del resultado (variables independientes) y el abandono del proceso de atención (variable dependiente), se utilizó el test de independencia Chi cuadrado $\left(\chi^{2}\right)$. La significación estadística fue establecida en 0,05. 
Figura 1. Mujeres con Papanicolaou con lesiones escamosas intraepiteliales de alto grado (HSIL) y carcinoma atendidas en el sistema público de la provincia de Jujuy, Argentina, 2010.

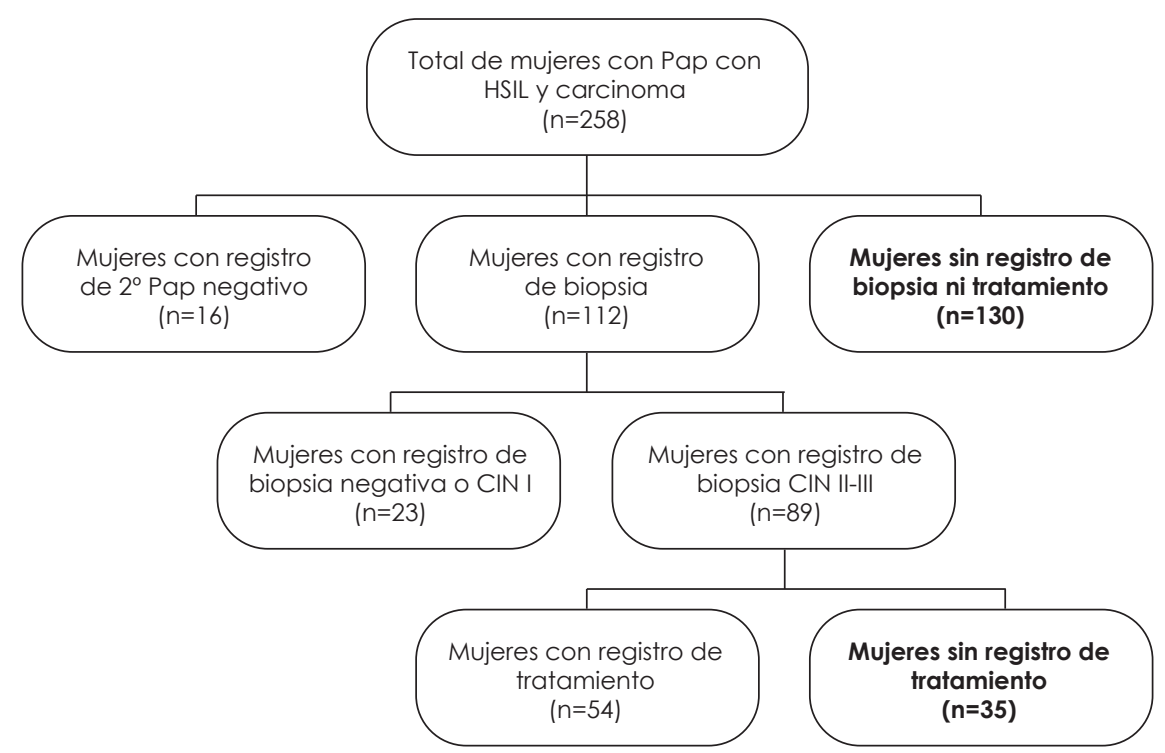

Fuente: Elaboración propia a partir de datos disponibles en el Sistema de Información para el Tamizaje (SITAM), bases de datos del Programa Provincial de Prevención y Control de Cáncer de Cuello Uterino y Cáncer de Mamas, registros de laboratorio de anatomía patológica y servicio de patología cervical del hospital de referencia.

$\mathrm{HSIL}=$ Lesiones escamosas intraepiteliales de alto grado .

\section{RESULTADOS}

\section{Magnitud del abandono}

Entre el 1 de enero y el 31 de diciembre de 2010, se registraron 21.304 Pap realizados en todo el sistema público de la provincia de Jujuy, de los cuales 297 eran Paps HSIL y carcinoma, correspondientes a 287 mujeres (10 mujeres registraban dos Pap en el período de referencia). 29 mujeres fueron excluidas debido a que registraban tratamientos en años anteriores (histerectomía, conización o LEEP), dejando una muestra total de 258 mujeres incluidas en el estudio.

Según las fuentes secundarias, 93 mujeres $(36 \%)$ con diagnóstico HSIL y carcinoma registraban información completa sobre biopsias y/o tratamiento, y 165 (64\%) no registraban esta información: no había registro sobre biopsias ni tratamiento de 130 mujeres y para 35 mujeres adicionales existía registro de biopsias (CIN II-III) pero no de tratamientos (Figura 1).
De estas 165 mujeres elegibles para realizar el cuestionario se contactaron 82 . Además se identificaron tres mujeres fallecidas y una mujer histerectomizada en el año 2008. No fue posible contactar a 79 mujeres debido a que sus direcciones estaban incompletas, eran erróneas o inexistentes. Todas las mujeres contactadas aceptaron participar del estudio. En total el estudio recabó información sobre 179 mujeres de las 258 diagnosticadas con HSIL y carcinoma durante 2010: 97 mujeres con registro de seguimiento y tratamiento (incluye las fallecidas) y 82 que fueron contactadas.

Del total de las 82 mujeres encuestadas, el $51,2 \%(n=42)$ abandonó el proceso de atención. El 48,8\% $(n=40)$ restante -que según los datos secundarios no poseían registro de biopsia y/o tratamiento- continuó con el proceso de atención. La mayoría de estas mujeres lo hizo en el sistema público, pero esta información no estaba registrada al momento de realizar la sistematización evidenciando retrasos en el registro. Solo 
Cuadro 1. Distribución porcentual del abandono del proceso de atención de las mujeres con lesiones escamosas intraepiteliales de alto grado (HSIL) y carcinoma según características sociodemográficas y económicas, uso del sistema de salud y región de residencia. Jujuy, Argentina, 2010.

\begin{tabular}{|c|c|c|c|c|c|c|}
\hline \multirow[t]{2}{*}{ Características } & \multicolumn{2}{|c|}{$\begin{array}{c}\text { Mujeres } \\
\text { contactadas }\end{array}$} & \multicolumn{4}{|c|}{$\begin{array}{c}\text { Abandono del proceso } \\
\text { de atención }\end{array}$} \\
\hline & Total & $\%$ & Sí & $\%$ & No & $\%$ \\
\hline Total & 82 & 100,0 & 42 & 100,0 & 40 & 100,0 \\
\hline \multicolumn{7}{|l|}{ Edad } \\
\hline $18-34$ & 27 & 32,9 & 13 & 31,0 & 14 & 35,0 \\
\hline $35-55$ & 37 & 45,1 & 19 & 45,2 & 18 & 45,0 \\
\hline 55 o más & 18 & 22,0 & 10 & 23,8 & 8 & 20,0 \\
\hline \multicolumn{7}{|l|}{ Nivel educativo } \\
\hline Primaria incompleta-completa & 26 & 31,7 & 15 & 35,7 & 11 & 27,5 \\
\hline Primaria completa/Secundaria incompleta & 36 & 43,9 & 19 & 45,2 & 17 & 42,5 \\
\hline Secundaria completa/Terciaria incompleta-completa & 20 & 24,4 & 8 & 19,0 & 12 & 30,0 \\
\hline \multicolumn{7}{|l|}{ Siłuación familiar } \\
\hline Con pareja e hijos a cargo & 43 & 52,4 & 21 & 50,0 & 22 & 55,0 \\
\hline Con pareja sin hijos a cargo & 8 & 9,8 & 6 & 14,2 & 2 & 5,0 \\
\hline Sin pareja con hijos a cargo & 25 & 30,5 & 13 & 31,0 & 12 & 30,0 \\
\hline Sin pareja sin hijos a cargo & 6 & 7,3 & 2 & 4,8 & 4 & 10,0 \\
\hline \multicolumn{7}{|l|}{ Situación ocupacional } \\
\hline Ocupada & 43 & 52,4 & 23 & 54,8 & 20 & 50,0 \\
\hline Ama de casa & 23 & 28,0 & 11 & 26,2 & 12 & 30,0 \\
\hline Inactiva & 16 & 19,5 & 8 & 19,0 & 8 & 20,0 \\
\hline \multicolumn{7}{|l|}{ Situación habitacional } \\
\hline Adecuada & 43 & 52,4 & 25 & 59,5 & 18 & 45,0 \\
\hline Inadecuada & 39 & 47,6 & 17 & 40,5 & 22 & 55,0 \\
\hline \multicolumn{7}{|l|}{ Cobertura de salud } \\
\hline Obra social & 19 & 23,2 & 8 & 19,0 & 11 & 27,5 \\
\hline Pública & 63 & 76,8 & 34 & 81,0 & 29 & 72,5 \\
\hline \multicolumn{7}{|l|}{ Frecuencia de visita al ginecólogo } \\
\hline Cada $3 / 6 / 12$ meses & 24 & 29,3 & 12 & 28,6 & 12 & 30,0 \\
\hline Solo en los embarazos & 10 & 12,2 & 3 & 7,1 & 7 & 17,5 \\
\hline No tiene frecuencia & 48 & 58,5 & 27 & 64,3 & 21 & 52,5 \\
\hline \multicolumn{7}{|l|}{ Región de residencia } \\
\hline Centro & 37 & 45,1 & 19 & 45,2 & 18 & 45,0 \\
\hline Ramal & 9 & 11,0 & 3 & 7,1 & 6 & 15,0 \\
\hline Valles & 18 & 21,9 & 9 & 21,4 & 9 & 22,5 \\
\hline Quebrada y Puna & 18 & 21,9 & 11 & 26,2 & 7 & 17,5 \\
\hline
\end{tabular}

Fuente: Elaboración propia.

Nota: El valor de p no fue significativo para ninguna de las características con relación al abandono del proceso de atención. 
ocho mujeres mencionaron haber continuado su atención en algún efector de salud privado.

Del total de las mujeres para las que el estudio recabó información $(n=179)$, el 23,5\% $(n=42)$ abandonó el proceso de atención.

Si estimamos el porcentaje de abandono considerando a las 79 mujeres que no pudieron ser contactadas, obtenemos al menos tres escenarios posibles: si asumimos que las 79 mujeres no contactadas continuaron el proceso de atención, el porcentaje de abandono sería del $16 \%$. Si consideramos que las 79 mujeres no contactadas tuvieron un comportamiento similar a las contactadas $(51,2 \%$ de abandono vs. $48,8 \%$ no abandono), el porcentaje de abandono podría estimarse en un $32 \%$. Si todas las mujeres no contactadas abandonaron el proceso de atención el porcentaje de abandono ascendería al 47\%.

\section{Características de las mujeres entrevistadas}

El Cuadro 1 muestra las características de las mujeres entrevistadas $(n=82)$. Si bien no se observan diferencias estadísticamente significativas entre ambos grupos (abandonos/no abandonos), entre las mujeres que abandonaron el proceso de atención se observa una mayor proporción de mujeres entre 35 y 55 años (45,2\%); con niveles educativos bajos $(35,7 \%)$ y medios $(45,2 \%)$, ocupadas $(54,8 \%)$, con cobertura pública de salud $(81,0 \%)$ y que mencionan no poseer una frecuencia establecida de visita al ginecólogo (64,3\%).

\section{Momento y motivos de abandono}

El 26,2\% de las mujeres que abandonaron el proceso de atención lo hizo en la etapa de tamizaje. El 57,1\% abandonó en la etapa de diagnóstico y el $16,7 \%$ realizó biopsia con confirmación de la presencia de lesión precancerosa (CIN II-III) pero no realizó tratamiento (Cuadro 2).

Al indagar acerca de los motivos de abandono (Cuadro 3), el 40,5\% de las mujeres mencionó algún motivo relacionado con la organización y funcionamiento del sistema de salud. Entre este grupo de mujeres, el 28,6\% mencionó motivos relacionados con la organización de los
Cuadro 2. Distribución porcentual según la etapa en la que se produce el abandono del proceso de atención de las mujeres con lesiones escamosas intraepiteliales de alto grado (HSIL) y carcinoma. Jujuy, Argentina, 2010.

\begin{tabular}{lcc}
\hline $\begin{array}{l}\text { Etapa en la que se } \\
\text { produce el abandono }\end{array}$ & \multicolumn{2}{c}{$\begin{array}{c}\text { Mujeres con HSIL y } \\
\text { carcinoma }\end{array}$} \\
\cline { 2 - 3 } & $\mathbf{n}$ & $\%$ \\
\hline Tamizaje & 11 & 26,2 \\
Diagnóstico & 24 & 57,1 \\
Tratamiento & 7 & 16,7 \\
Total & 42 & 100,0
\end{tabular}

Fuente: Elaboración propia.

HSIL = Lesiones escamosas intraepiteliales de alto grado

servicios: retrasos en la obtención de los resultados, problemas para acceder a los turnos o largos tiempos de espera para ser atendidas; y el 11,9\% mencionó motivos relacionados al proceso de atención (problemas en la comunicación del resultado o malas experiencias de atención).

El 30,9\% de las mujeres que abandonó el proceso de atención, reportó motivos subjetivos: miedo, negación a la enfermedad o no considerar necesario continuar con la atención. Otros motivos mencionados como principal causa de abandono

Cuadro 3. Distribución porcentual de los motivos de abandono del proceso de atención mencionados por las mujeres con lesiones escamosas intraepiteliales de alto grado (HSIL) y carcinoma. Jujuy, Argentina, 2010.

\begin{tabular}{ccc}
\hline Motivos de abandono & $\begin{array}{c}\text { Mujeres con HSIL y } \\
\text { carcinoma }\end{array}$ \\
\cline { 2 - 3 } & $\mathrm{n}$ & $\%$ \\
\hline
\end{tabular}

$\begin{array}{lcc}\text { Relativos al sistema de salud } & & \\ \quad \text { Organización de los servicios } & 12 & 28,6 \\ \quad \text { Proceso de atención } & 5 & 11,9 \\ \text { Subjetivos } & 13 & 30,9 \\ \text { Personales/familiares } & 3 & 7,1 \\ \text { Laborales } & 2 & 4,8 \\ \text { Carga de trabajo doméstico } & 2 & 4,8 \\ \text { Traslado } & 2 & 4,8 \\ \text { No menciona motivo } & 3 & 7,1 \\ \text { Total } & 42 & 100,0\end{array}$

Fuente: Elaboración propia.

HSIL = Lesiones escamosas intraepiteliales de alto grado . 
fueron: problemas familiares $(7,1 \%)$, razones laborales $(4,8 \%)$, carga de trabajo doméstico y cuidado de niños $(4,8 \%)$ y barreras para el traslado $(4,8 \%)$.

Luego de la visita de la encuestadora, el $55 \%$ de las mujeres que había abandonado el proceso de atención retornó a la consulta entre los 10 y 30 días posteriores a la entrevista.

\section{Comunicación de los resultados}

Aunque en la mayoría de las variables relativas a la calidad en la entrega de los resultados no se observan diferencias estadísticamente significativas entre los grupos, las mujeres que abandonaron el proceso de atención reportaron en menor proporción indicadores de buena calidad en la entrega de resultados que aquellas que no abandonaron. El $42,1 \%$ de las mujeres que abandonaron y el $57,9 \%$ de las que no abandonaron mencionan que algún profesional del sistema de salud la llamó o la visitó en su casa para entregarle el resultado o citarla a una consulta en donde se le entregaría el resultado (Cuadro 4).

Para el análisis de la calidad en la entrega de resultados, se excluyeron las mujeres que no lo conocían $(n=11)$. Entre las mujeres que sí conocían el resultado $(n=71)$, el $38,6 \%$ de las que abandonaron y el $61,4 \%$ de las que no abandonaron

Cuadro 4. Distribución porcentual del abandono del proceso de atención según indicadores de calidad percibida por las mujeres con lesiones escamosas intraepiteliales de alto grado (HSIL) y carcinoma, en la entrega de los resultados. Jujuy, Argentina, 2010.

\begin{tabular}{|c|c|c|c|c|c|c|}
\hline \multirow[t]{2}{*}{ Indicadores de calidad percibida } & \multicolumn{2}{|c|}{$\begin{array}{l}\text { Mujeres } \\
\text { contactadas }\end{array}$} & \multicolumn{4}{|c|}{$\begin{array}{c}\text { Abandono del proceso } \\
\text { de atención }\end{array}$} \\
\hline & Total & $\%$ & Sí & $\%$ & No & $\%$ \\
\hline \multicolumn{7}{|c|}{$\begin{array}{l}\text { ¿Algún profesional la visitó/la llamó para entregarle } \\
\text { resultado? }\end{array}$} \\
\hline Sí & 38 & 100,0 & 16 & 42,1 & 22 & 57,9 \\
\hline No & 44 & 100,0 & 26 & 59,0 & 18 & 41,0 \\
\hline Total & 82 & 100,0 & 42 & 51,2 & 40 & 48,8 \\
\hline \multicolumn{7}{|l|}{ Tiempo para recibir el resultado* } \\
\hline 1 a 4 semanas & 44 & 100,0 & 21 & 47,7 & 23 & 52,3 \\
\hline 5 semanas o más & 27 & 100,0 & 10 & 37,0 & 17 & 63,0 \\
\hline Total & 71 & 100,0 & 31 & 43,7 & 40 & 56,3 \\
\hline \multicolumn{7}{|c|}{ ¿Le explicaron lo que significaba el resultado?* } \\
\hline Sí & 57 & 100,0 & 22 & 38,6 & 35 & 61,4 \\
\hline No & 14 & 100,0 & 9 & 64,3 & 5 & 35,7 \\
\hline Total & 71 & 100,0 & 31 & 43,7 & 40 & 56,3 \\
\hline \multicolumn{7}{|c|}{ ¿Le explicaron cuáles eran los pasos posteriores a seguir?* } \\
\hline Sí & 33 & 100,0 & 11 & 33,3 & 22 & 66,7 \\
\hline No & 38 & 100,0 & 20 & 52,6 & 8 & 47,4 \\
\hline Total & 70 & 100,0 & 31 & 43,7 & 40 & 56,3 \\
\hline \multicolumn{7}{|c|}{ ¿El médico le preguntó si tenía alguna consulta, duda?*** } \\
\hline Sí & 21 & 100,0 & 4 & 19,0 & 17 & 81,0 \\
\hline No & 40 & 100,0 & 19 & 47,5 & 21 & 52,5 \\
\hline Total & 61 & 100,0 & 23 & 37,7 & 38 & 62,3 \\
\hline
\end{tabular}

Fuente: Elaboración propia.

Nota: El valor de p solo fue significativo para el indicador de calidad percibida referente a si el médico le preguntó si tenía alguna consulta, duda $(p=0,029)$.

*Fueron excluidas 11 mujeres que no conocían el resultado.

**Fueron excluidas 11 mujeres que no conocían el resultado y 10 casos perdidos. 
mencionó que el médico les explicó lo que significaba el resultado. El 33,3\% de las que abandonaron y el $66,7 \%$ de las que no abandonaron consideró que el médico les explicó adecuadamente cuál era el siguiente paso que debían realizar. La pregunta acerca de si el médico le preguntó si tenía alguna duda sobre el resultado o los pasos a seguir fue contestada por 61 mujeres que conocían el resultado. Entre ellas, el 19,0\% de las mujeres que abandonaron y el $81,0 \%$ de las que no abandonaron contestó afirmativamente $(p=0,029)$.

\section{DISCUSIÓN}

Según nuestro conocimiento este es el primer estudio que analiza, para un año dado, la magnitud y los motivos de abandono del proceso de seguimiento y tratamiento de la totalidad de mujeres con Pap HSIL y carcinoma atendidas en el sistema público de una provincia argentina con altas tasas de mortalidad por cáncer de cuello uterino.

En nuestro estudio el porcentaje de abandono fue del $23 \%$, con una posible fluctuación entre $16 \%$ y $47 \%$ según se estime el comportamiento de las mujeres que no fueron contactadas. Las investigaciones realizadas en EE.UU. y Europa muestran tasas de abandono entre 10\% y 45\% (18,23-25). En los países de América Latina, en donde los programas de prevención de cáncer de cuello uterino no han alcanzado los resultados esperados (26) estos porcentajes son mayores: entre $20 \%$ y $75 \%(13,17,22,27-30)$. Si bien las comparaciones deben realizarse con precaución -debido a las diferencias en los sistemas de salud, las poblaciones incluidas en los estudios y las definiciones de abandono- el porcentaje de abandono estimado para la provincia de Jujuy es similar a los reportados en estudios realizados en México, Colombia y Brasil: $20 \%$, 27\% y $29 \%$ respectivamente $(22,28,29)$.

Dado el impacto que tiene el adecuado seguimiento y tratamiento en la reducción de la mortalidad por cáncer de cuello uterino (10), la disminución de los porcentajes de abandono se presenta como un desafío para todos los programas de prevención de cáncer de cuello uterino en América Latina. La mayoría de estos programas han priorizado la realización de acciones tendientes a lograr altas coberturas de tamizaje, pero no demuestran esfuerzos similares para el logro del adecuado diagnóstico y tratamiento de las mujeres con lesiones precancerosas (31). En el contexto actual de la incorporación de nuevas tecnologías para el tamizaje (32), sea cual fuere el test de tamizaje utilizado, es necesario que los sistemas de salud estén articulados y que el adecuado diagnóstico y tratamiento de las mujeres con lesiones precancerosas esté garantizado.

En nuestro estudio, la mayoría de los abandonos ocurrieron en la etapa de diagnóstico, es decir, luego de recibir el resultado y antes de acceder a la biopsia. Estos resultados son consistentes con otras investigaciones $(22,28)$ y pueden ser considerados indicadores de falencias en el traspaso de información y responsabilidades entre los servicios de tamizaje y los de diagnóstico y tratamiento, relevadas en diversos estudios $(20,27)$. La trayectoria de atención de una mujer que es derivada para realizar una biopsia sale del ámbito del profesional que realizó el tamizaje, quien muchas veces desconoce el resultado final del proceso (12). La identificación de profesionales responsables del seguimiento de las mujeres con HSIL y carcinoma en cada región, la implementación de sistemas de aviso/alarma frente a estos casos y la reformulación del sistema de referencia y contrarreferencia, son estrategias posibles para mejorar la trayectoria de las mujeres entre las distintas instituciones involucradas y para evitar los abandonos en momentos claves del proceso. A su vez, estas estrategias requieren de un sistema de información que permita el monitoreo del proceso. La ausencia de registros sistemáticos sobre seguimiento y tratamiento es un problema que ha sido reportado tanto en América Latina $(27,31)$ como en Argentina $(12,33)$. El hecho de que al inicio del proyecto el sistema de salud no contara con información acerca de lo ocurrido con el diagnóstico y tratamiento del $64 \%$ de las mujeres con Pap HSIL y carcinoma, reveló falencias en el registro de la información que se estaba implementando hasta el momento. Los resultados de la investigación se discutieron en reuniones con las autoridades y efectores locales en donde se identificaron los obstáculos para el registro y se trabajó sobre cómo mejorar la carga de datos y el uso de la información provista por el SITAM como paso importante para la reducción de los abandonos. 
En cuanto a los motivos de abandono, en primer lugar las mujeres mencionan aspectos relacionados con la organización de los servicios de salud. Los obstáculos institucionales que ocasionan el abandono de las mujeres son similares a los reportados por investigaciones en América Latina $(12,13,22,27,28)$. Los resultados relativos a estos obstáculos fueron trabajados conjuntamente con las autoridades sanitarias de la provincia con el objetivo de reformular algunos aspectos de la organización del proceso de tamizaje-seguimiento-tratamiento. Entre las modificaciones producidas se destacan el aumento de la disponibilidad de turnos para las biopsias de mujeres con HSIL y carcinoma la reorganización de las redes de derivación en las distintas regiones de la provincia y la implementación desde julio de 2011 del proyecto "Navegadoras", impulsado y financiado por el Ministerio de Salud de la Nación. Dicho proyecto permite contar con recursos humanos cuyo rol principal es brindar apoyo y acompañamiento a las mujeres que necesitan diagnóstico y tratamiento para reducir las barreras sociales e institucionales de acceso a la atención.

En segundo lugar, las mujeres mencionan motivos de abandono subjetivos. Estos resultados coinciden con la vasta evidencia acerca de que las percepciones y conocimientos de las mujeres en torno al cáncer de cuello uterino, las formas de prevención y tratamientos son factores que condicionan las prácticas con relación a la enfermedad (13,22,28,34-36), ya sea produciendo el abandono del seguimiento y tratamiento $(22,28,34)$ o actuando como barrera para la realización del Pap $(35,36)$. Estos resultados reflejan la importancia de considerar los aspectos simbólicos de la enfermedad al momento de plantear las estrategias de salud pública tendientes a reducir las tasas de abandono. Los motivos por los cuales las mujeres utilizan o no los servicios estarán supeditados a las percepciones acerca de su salud, sus dolencias y la existencia o no de soluciones para sus problemas (37). Eliminar las barreras subjetivas para la atención requiere de estrategias integrales que incluyan espacios de consejería en donde las necesidades de las mujeres sean escuchadas e incorporadas en las estrategias para la resolución del problema.
Los resultados muestran que las mujeres que han abandonado el proceso de atención mencionan en menor proporción indicadores de buena calidad en la entrega de resultados que aquellas que no han abandonado. Si bien no se observan diferencias estadísticamente significativas entre los grupos (quizá debido al tamaño de la muestra utilizada) su coincidencia con investigaciones que muestran la influencia de la inadecuada comunicación entre los profesionales y las mujeres en los abandonos del proceso de seguimiento y tratamiento $(30,38-42)$, permiten pensar que la entrega de los resultados es un elemento clave para el análisis de los abandonos. Diversos estudios han mostrado que las dificultades en la relación entre profesionales y mujeres producen, en algunos casos, que no se interprete adecuadamente el significado de un Pap anormal (39-42) o que se generen situaciones de estrés y ansiedad que actúan como barreras para la continuidad del tratamiento (40). A pesar de que las mujeres que abandonaron el tratamiento reportan menor calidad en la entrega de resultados, solo cuatro mujeres mencionan como motivo de abandono problemas en la comunicación de los resultados. Si bien es necesario profundizar este tema en futuras investigaciones, esto podría explicarse por la naturalización de las características de los procesos de atención -la relación médico-paciente asimétrica, la desvalorización de todo saber diferente al biomédico (43)-, que ubican muchas veces a las mujeres en un lugar de pacientes-pasivas, y que pueden actuar como barreras para la atención, aunque no sean explicitadas como tales. Estudios realizados a partir de observaciones en la consulta médica muestran que cuando se abre un espacio para la dimensión emocional y la escucha atenta, se produce una comunicación más próxima entre profesionales y usuarios y se logran mejores resultados en el proceso de atención $(44,45)$. La constitución de espacios específicos para la entrega de resultados, diseñados acorde a las necesidades de las mujeres, puede contribuir a reducir las situaciones de estrés o ansiedad. La constitución de estos espacios requiere de un proceso de capacitación específica sobre competencias y técnicas adecuadas para la comunicación de los resultados.

Existen algunas limitaciones en nuestro estudio que deben considerarse. No fue posible 
obtener información para el 30,6\% $(n=79)$ del total del universo de mujeres con diagnóstico de HSIL y carcinoma $(n=258)$. Este hecho podría producir una subestimación del porcentaje de abandono, es por eso que en los resultados se calculó la posible fluctuación del abandono (entre $16 \%$ y $47 \%$ ) según se estime el comportamiento de las mujeres que no fueron contactadas. Otra limitación del estudio es que no se incluyeron en el análisis de las diferencias entre el grupo de mujeres que abandonaron y las que no abandonaron, a las 93 mujeres con registros sobre seguimiento y tratamiento. Esta decisión metodológica fue tomada sobre la base de la dificultad logística que entraña la búsqueda de mujeres residentes en distintas áreas y localidades de la provincia. Dado que los recursos disponibles para la investigación eran limitados, se decidió priorizar la búsqueda de mujeres sin registro de seguimiento y tratamiento, asumiendo que eran las más vulnerables frente a la enfermedad y las que mayor beneficio obtendrían del contacto con la entrevistadora. Tampoco se incluyó en este análisis a las 79 mujeres sin registro y que no pudieron ser contactadas por errores en los datos de contacto. Si consideramos el registro de la información como un indicador de una mejor calidad en el proceso de atención de salud (46), entonces es probable que el análisis esté subestimando las diferencias en calidad de entrega de resultados encontradas entre las mujeres que abandonaron y las que no abandonaron. Si esta hipótesis se cumple, reforzaría aún más la influencia de la inadecuada comunicación entre los profesionales y las mujeres en los abandonos del proceso de seguimiento y tratamiento detectada por nuestro estudio. Es necesario desarrollar nuevos estudios que indaguen en profundidad este tema.

\section{CONCLUSIÓN}

Los resultados de esta investigación han permitido evidenciar un problema que hasta el momento estaba invisibilizado en la agenda de salud pública provincial, e introducir modificaciones en los servicios involucrados en prevención del cáncer de cuello uterino. Si bien esto no ha eliminado totalmente los obstáculos para la atención, ha sido un primer paso necesario para la reducción de los porcentajes de abandono y de las barreras para la accesibilidad a la atención de la salud de las mujeres. El hecho de que más de la mitad de los abandonos contactados hayan retornado al sistema de salud inmediatamente después de la entrevista da indicios de que las acciones que se están emprendiendo en la provincia pueden dar buenos resultados en el corto y mediano plazo.

\section{AGRADECIMIENTOS}

Las autoras agradecen el financiamiento de la Organización Panamericana de la Salud que hizo posible este estudio. Agradecemos especialmente a la Dra. Alicia Campanera (Programa Provincial de Detección Precoz de Cáncer de Cuello Uterino), al Dr. Vicente Corte y a la Dra. Susana Beguier (Dirección Provincial de Maternidad e Infancia), a la Lic. Adriana Magdaleno (Dirección Provincial de APS y Acción Comunitaria), al Dr. Oscar Marín (Jefe del Servicio de Anatomía Patológica del Hospital Pablo Soria) y la Dra. Cristina Falcón (Servicio de Patología Cervical del Hospital Pablo Soria). Además agradecemos a Eva Arroyo y Cecilia Sandoval por la realización de las encuestas. Finalmente, un especial agradecimiento a las mujeres que generosamente brindaron su tiempo para contestar las entrevistas.

\section{CONFLICTOS DE INTERESES}

No hay conflictos de interés por parte de las autoras del trabajo. Las opiniones vertidas corresponden a las autoras y no representan la visión de la entidad que ha financiado el proyecto. 


\section{NOTAS FINALES}

a. El test de Papanicolaou (o citología convencional) permite identificar lesiones en el cuello del útero en la etapa preclínica (lesiones precancerosas). La lenta progresión de la enfermedad permite que, si la lesión es detectada, pueda ser tratada antes de convertirse en cáncer. Según la literatura especializada, un programa organizado de tamizaje se define como un sistema integrado en el cual las mujeres son invitadas, se les realiza un test de tamizaje, reciben los resultados (en tiempo y forma), son derivadas para tratamiento si es necesario y vuelven para repetir el tamizaje según la recomendación. El objetivo final del programa de prevención es la reducción de la incidencia y mortalidad por cáncer de cuello de útero. Para ello, un programa debe cumplir con tres condiciones básicas: a) la alta cobertura de la población objetivo, b) el tamizaje con un test de buena calidad, y c) el adecuado tratamiento y seguimiento de las mujeres con lesiones precancerosas y cáncer.

b. La prevención mediante las estrategias de tamizaje se diferencia del diagnóstico, constituyendo dos etapas de un mismo proceso. El tamizaje se orienta hacia sujetos considerados "sanos" para, entre ellos, identificar un relativamente pequeño número de personas que deben realizar exámenes

\section{REFERENCIAS BIBLIOGRÁFICAS}

1. Anttila A, Nieminen P. Cervical cancer screening programme in Finland. European Journal of Cancer. 2000;36(17):2209-2214.

2. Zur Hausen H. Papillomavirus infection: a major cause of human cancers. Biochimica et Biophysica Acta. 1996;1288(2):F55-F78.

3. Bosch FX, Manos MM, Muñoz N, Sherman M, Jansen A, Peto J, Schiffman MH, Moreno V, Kurman R, Shah KV. The IBSCC study group: Prevalence of human papillomavirus in cervical cancer: a worldwide perspective. Journal of the National Cancer Institute. 1995;87(11):796-802.

4. Herrero R, Ferreccio C, Salmerón J, Almonte M, Sánchez GI, Lazcano-Ponce E, Jerónimo J. New approaches to cervical cancer screening in Latin America and the Caribbean. Vaccine. 2008;26(Suppl 11):L49-L58.

5. Cuzick J, Harbin M, Sankaranarayanan R, Tsu V, Ronco G, Mayrand ME, Dillner J, Meijer C. Overview of human papillomavirus-based and complementarios de detección para definir si realmente poseen una lesión pre-maligna o cáncer.

c. La escisión electroquirúrgica con asa (LEEP por sus siglas en inglés) es un procedimiento relativamente sencillo para el tratamiento de las lesiones precancerosas, en el que se utiliza un hilo conductor delgado en forma de asa para remover el área anormal del cuello del útero.

d. El diseño metodológico utilizado en este estudio, así como sus resultados, se enmarcan en el Proyecto de Tesis de Doctorado en Ciencias Sociales (Universidad de Buenos Aires) de la Lic. Melisa Paolino, dirigido por la Dra. Silvina Arrossi. Una adaptación de este protocolo también es utilizado en un proyecto actualmente en curso en el Municipio de Florencio Varela, Buenos Aires (Proyecto PICT-2008-1545) financiado por la Agencia Nacional de Promoción Científica y Tecnológica, Fondo para la Investigación Científica y Tecnológica.

e. Desde el 2011 el Instituto Nacional del Cáncer y el Programa Nacional de Prevención del Cáncer Cérvico-uterino, junto al Ministerio de Salud de Jujuy están implementando un proyecto demostración que incorpora el test del virus del papiloma humano (VPH) como test de tamizaje primario en toda la provincia (14).

other novel options for cervival cancer screening in developed and developing countries. Vaccine. 2008;26(10):K29-K41.

6. Gage J, Castle P. Preventing cervical cancer globally by acting locally: if not now, when? Journal of the National Cancer Institute. 2010;102(20):1524-1527.

7. Sanjosé S, Bosch FX, Muñoz N, Tafur L, Gili M, Izarzugaza I, Izquierdo A, Navarro C, Vergara A, Muñoz MT, Ascunce $\mathrm{N}$, Shah KV. Socioeconomic differences in cervical cancer: two case-control studies in Colombia and Spain. American Journal of Public Health. 1996;86(11):1532-1538.

8. Parikh S, Brennan P, Boffetta P. Meta-analysis of social inequality and the risk o cervical cancer. International Journal of Cancer. 2003;105(5):687691.

9. Ministerio de Salud. Programa Nacional de Prevención de Cáncer Cérvico-uterino [Internet]. Buenos Aires: Ministerio de Salud [citado 15 feb 2012] Disponible en: http://www.msal.gov.ar/cancer-cervico-uterino/. 
10. Gamboa O, Chicaíza L, García-Molina M, Díaz J, González M, Murillo R, Ballesteros $M$, Sánchez R. Cost-effectiveness of conventional cytology and HPV DNA testing for cervical cancer screening in Colombia. Salud Pública de México. 2008;50(4):276-285.

11. Arrossi S, Paolino M. Proyecto para el mejoramiento del Programa Nacional de Prevención de Cáncer de Cuello Uterino: Informe final: Diagnóstico de situación del Programa Nacional y Programas. Buenos Aires: OPS; 2008.

12. Paolino M, Pantelides EA, Bruno M, Maceira V, Peña L, Godoy J, Farao S, Arrossi S. Determinantes sociales del seguimiento y tratamiento de mujeres con Pap anormal en Avellaneda, Provincia de Buenos Aires. Revista Argentina de Salud Pública. 2011;2(8):21-27.

13. Ramos S, Pantelides EA. Prevención secundaria del cáncer de cuello de útero: determinantes de la deserción de pacientes. Cuadernos Médicos Sociales. 1990;53:37-50.

14. Ministerio de Justicia y Derechos Humanos. Resolución 1472/2011, "Proyecto Demostración para la incorporación de la Prueba de HPV como Tamizaje Primario". Boletín Oficial de la República Argentina [Internet]. 2011 [citado 15 feb 2012];119(32.242). Disponible en: http://www.boletinoficial.gov.ar/Inicio/index.castle?s $=1 \&$ fea $=26 / 09 / 2011$

15. Solomon D, Nayar D. The Bethesda System for reporting cervical cytology: definitions, criteria, and explanatory notes. New York: Sprinter; 2004.

16. World Health Organization. Comprensive Cancer Control. A guide to essencial practice. Ginebra: WHO; 2006.

17. Gage J, Ferreccio C, Gonzales M, Arroyo R, Huivín M, Robles S. Follow-up care of women with an abnormal cytology in a low-resource setting. Cancer Detection and Prevention. 2003;27(6):466-471.

18. Monnet E, Marquant A, Genin P, Mauny F, Carbillet JP. Quality of follow-up of women with high grade squamous intra-epithelial lesion (HGSIL) cervical smears: results from a population-based organised screening programme. European Journal of Obstetrics, Gynecology and Reproductive Biology. 2004;113(2):234-239.

19. Instituto Nacional de Estadísticas y Censos. Glosario. [Internet]. Buenos Aires: INDEC [citado 18 feb 2012]. Disponible en http://www.indec. gov.ar/glosario/textos_glosario.asp?id=20.
20. Zapka J, Taplin S, Anhang Price R, Cranos C, Yabroff R. Factors in quality care - The case of follow-up to abnormal cancer screening tests Problems in the steps and interfaces of care. Journal of the National Cancer Institute Monographs. 2010;2010(40):58-71.

21. Donabedian A. Garantía y monitoría de calidad de la atención médica. México: Instituto Nacional de Salud Pública; 1990.

22. Wiesner C, Cendales R, Murillo R, Piñeiros S, Tovar S. Seguimiento de mujeres con anormalidad citológica de cuello uterino, en Colombia. Revista de Salud Pública. 2010;12(1):1-13.

23. Spence A, Goggin P, Franco EL. Process of care failures in invasive cervical cancer: Systematic review and meta-analysis. Preventive Medicine. 2007;45(2-3):93-106.

24. Cardin V, Grimes R, Jiang Z, Pomeroy N, Harrell L, Cano P. Low-income minority women at risk for cervical cancer: a process to improve adherence to follow-up recommendations. Public Health Reports. 2001;116(6):608-616.

25. Leyden W, Manos M, Geiger A, Weinmann $S$, Mouchawar J, Bischoff K. Cervical cancer in women with comprehensive health care access: attributable factors in the screening process. Journal of National Cancer Institute. 2005;97(9):675-683.

26. Sankaranarayanan R, Budukh AM, Rajkumar $R$. Effective screening programmes for cervical cancer in low- and middle-income developing countries. Bulletin of the World Health Organization. 2001;79(10):954-962.

27. Dzuba IG, Calderon R, Bliesner S, Luciani S, Amado F, Jacob M. A participatory assessment to identify strategies for improved cervical cancer prevention and treatment in Bolivia. Revista Panamericana de Salud Pública. 2005;18(1):53-63.

28. Hernández-Alemán FR, Ornelas-Bernal LA, Apresa-García T, Sánchez-Garduno S, MartínezRodríguez OA, Hernández-Hernández DM. Motivos de abandono en el proceso de atención médica de lesiones precursoras de cáncer cervicouterino. Revista de Investigación Clínica. 2006;58(3):217-227.

29. Nascimento MI, Koifman RJ, Mattos IE, Monteiro GTR. Predictors of non-adherence to the prescribed follow-up in women with highgrade squamous intraepithelial lesion (HSIL). Saúde e Sociedade. 2009;18(2):325-333. 
30. Jeong SJ, Saroha E, Knight J, Roofe M, Jolly PE. Determinants of adequate follow-up of an abnormal Papanicolaou result among Jamaican women in Portland, Jamaica. Cancer Epidemiology. 2011;35(2):211-216.

31. Murillo R, Almonte M, Pereira A, Ferrer E, Gamboa OA, Jerónimo J, Lazcano-Ponce E. Cervical cancer screening programs in Latin America and the Caribbean. Vaccine. 2008;26(Suppl 11):L37-L48.

32. Almonte M, Murillo R, Sánchez GI, Jerónimo J, Salmerón J, Ferreccio C, Lazcano-Ponce E, Herrero R. Nuevos paradigmas y desafíos en la prevención y control del cáncer de cuello uterino en América Latina. Salud Pública de Mexico. 2010;52(6):544-559.

33. Arrossi S, Paolino M, Sankaranarayanan R. Challenges faced by cervical cancer prevention programs in developing countries: a situational analysis of program organization in Argentina. Revista Panamericana de Salud Pública. 2010;28(4):249-257.

34. Sharpe $P$, Brandt $H$, Mccree D. Knowledge and beliefs about abnormal Pap test results and HPV among women with high-risk HPV: Results from in-depth interviews. Women \& Health. 2005;42(2):107-133.

35. Agurto I, Bishop A, Sanchez G, Betancourt Z, Robles S. Perceived barriers and benefits to cervical cancer screening in Latin America. Preventive Medicine. 2004;39(1):91-98.

36. Zamberlin N, Thouyaret L, Arrossi S. Lo que piensan las mujeres: conocimientos y percepciones sobre cáncer de cuello de útero y realización de PAP. Buenos Aires: OPS; 2011.

37. Menéndez E. El punto de vista del actor: Homogeneidad, diferencia e historicidad. México DF: CIESAS; 1994.

38. Zeisler H, Mayerhofer K, Joura E, Sator M, Kainz C. Psychological burder or woman with mild cervical intraepithelial neoplasia. Oncology Report. 1997;4(5):1063-1065.
39. Zapka J, Puleo E, Taplin S, Goins K, Ulcickas $\mathrm{Y}$, Mouchawar J. Processes of care in cervical and breast cancer screening and follow-up: the importance of communication. Preventive Medicine. 2004;39(1):81-90.

40. Castro-Vásquez MC, Arellano-Gálvez MC. Acceso a la información de mujeres con VPH, displasia y cáncer cervical in situ. Salud Pública de México. 2010;52(3):207-212.

41. Kavanagh A, Broom D. Women's understanding of abnormal cervical smear test results: A qualitative interview study. British Medical Journal. 1997;314(7091):1388-1391.

42. McKee M, Lurio J, Marantz P, Burton W, Mulvihill M. Barriers to follow-up of abnormal Papanicolaou smears in an urban community health center. Archive of Family Medicine. 1999;8(2):129-134.

43. Menéndez E. Modelos de atención de los padecimientos: de exclusiones teóricas y articulaciones prácticas. En: Spinelli $\mathrm{H}$, compilador. Salud colectiva: Cultura, instituciones y subjetividad; Epidemiología, gestión y políticas. Buenos Aires: Lugar Editorial; 2004. p. 11-47.

44. Barry CA, Stevenson FA, Britten N, Barber N, Bradley CP. Giving voice to the lifeworld: More humane, more effective medical care? A qualitative study of doctor-patient communication in general practice. Social Science and Medicine. 2001;53(4):487-505.

45. Bonet O. Emociones e sofrimentos nas consultas médicas: Implicações da sua irrupção. Teoria e Cultura. 2006;1(1):117-138.

46. Jiménez-Paneque RE. Indicadores de calidad y eficiencia de los servicios hospitalarios: Una mirada actual. Revista Cubana de Salud Pública [Internet]. 2004 [citado 2012 Ago 15]; 30(1): Disponible en: http://scielo.sld.cu/scielo.php? script $=$ sci arttext \&pid $=$ S0864-34662004 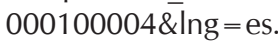

\section{FORMA DE CITAR}

Paolino M, Arrossi S. Análisis de los motivos del abandono del proceso de seguimiento y tratamiento por parte de mujeres con lesiones precursoras de cáncer de cuello uterino en la provincia de Jujuy: implicancias para la gestión. Salud Colectiva. 2012;8(3):247-261.

Recibido el 8 de mayo de 2012

Versión final presentada el 28 de agosto de 2012

Aprobado el 28 de octubre de 2012 